\title{
ON MODULI OF CONTINUITY AND DIVERGENCE OF FOURIER SERIES ON GROUPS
}

\author{
C. W. ONNEWEER
}

ABstract. Let $G$ be a 0 -dimensional, metrizable, compact, abelian group. Then its character group $X$ is a countable, discrete, torsion, abelian group. N. Ja. Vilenkin defined an enumeration for the elements of $X$ and developed part of the Fourier theory on $G$. Among other things he proved on $G$ a theorem similar to the DiniLipschitz test for trigonometric Fourier series. In this note we will show that Vilenkin's result is in some sense the best possible by proving the existence of a continuous function $f$ on $G$ whose modulus of continuity, $\theta_{k}(f)$, satisfies $\theta_{k}(f)=O\left(k^{-1}\right)$ as $k \rightarrow \infty$ and whose Fourier series diverges at a point of $G$. The function $f$ will be defined by means of the analogue in $X$ of the classical Fejér polynomials.

Throughout this paper we will use the terminology and notations of [2] or [4] and we will assume that the reader is familiar with at least one of these papers.

Let $G$ and $X$ be as in the abstract. The best-known example of such a group $G$ is $\prod_{n=1}^{\infty}(Z(2))_{n}$, which has the system of Walsh functions as its dual group (see [1]). Vilenkin's theorem, mentioned in the abstract, is the following $[4,3.5]$ : If $G$ is a primary group and if $f$ is a continuous function on $G$ whose modulus of continuity, $\theta_{k}(f)$, satisfies $\theta_{k}(f)=o\left(k^{-1}\right)$ as $k \rightarrow \infty$, then the Fourier series of $f$ converges uniformly. In [2, Corollary 2] Onneweer and Waterman obtained the same result for groups $G$ which satisfy the condition that $\sup _{n} p_{n}$ $=p<\infty$. From now on we will only consider groups $G$ for which $\sup _{n} p_{n}=p<\infty$. We establish the following theorem.

TheOREM. There exists a continuous function $f$ on $G$ such that (i) the Fourier series of $f$ does not converge at $0 \in G$ and (ii) $\theta_{k}(f)=O\left(k^{-1}\right)$ as $k \rightarrow \infty$.

The proof resembles G. Faber's proof of a similar theorem for trigonometric Fourier series [5, p. 302]. It will be preceded by two lemmas.

LEMMA 1. Let $a_{0}=1$ and if $n$ satisfies $m_{k} \leqq n<m_{k+1}$ for some $k$, let $a_{n}=(-1)^{k}\left(m_{k+1}-m_{k}\right)^{-1}$. Let $S(x)=\sum_{n=0}^{\infty} a_{n} \chi_{n}(x)$.

Received by the editors February 25, 1970.

AMS 1970 subject classifications. Primary 42A56; Secondary 43A75.

Key words and phrases. 0-dimensional group, metrizable group, compact abelian group, Fourier series, Walsh functions, modulus of continuity, Dini-Lipschitz test, Fejér polynomials. 
Then the partial sums of $S(x), S_{m}(x ; S)=\sum_{n=0}^{m-1} a_{n} \chi_{n}(x)$, satisfy

$$
\left|S_{m}(x ; S)\right| \leqq 4
$$

uniformly in $m$ and in $x \in G$.

Proof. Let $m$ be any natural number and take $s$ so that $m_{s}<m$ $\leqq m_{s+1}$. Set $m=b_{s} m_{s}+m^{\prime}$, with $0<b_{s} \leqq p_{s+1}$ and $0 \leqq m^{\prime}<m_{s}$. Then

$$
\begin{aligned}
S_{m}(x ; S) & =\sum_{i=0}^{m-1} a_{i} \chi_{i}(x) \\
& =1+\sum_{k=0}^{8-1} \frac{(-1)^{k}}{m_{k+1}-m_{k}} \sum_{i=m_{k}}^{m_{k+1}-1} \chi_{i}(x)+\sum_{i=m_{i}}^{m-1} \frac{(-1)^{*}}{m_{s+1}-m_{i}} \chi_{i}(x) \\
& =1+B_{1}+B_{2} .
\end{aligned}
$$

Next we observe that, for each $k$,

$$
\begin{aligned}
\sum_{i=m_{k}}^{m_{k+1}-1} \chi_{i}(x) & =\sum_{j=1}^{p_{k+1}-1} \sum_{i=j m_{k}}^{(j+1) m_{k}-1} \chi_{i}(x)=\sum_{j=1}^{p_{k+1}-1} \sum_{i=0}^{m_{k}-1} \chi_{j m_{k}+i}(x) \\
& =\sum_{j=1}^{p_{k+1}-1} \chi_{m_{k}}^{j}(x) \sum_{i=0}^{m_{k}-1} \chi_{i}(x)=\sum_{j=1}^{p_{k+1}-1} \chi_{m_{k}}^{j}(x) D_{m_{k}}(x) .
\end{aligned}
$$

Also, according to $[4,2.2]$ we have, for each $r$,

(i) if $x \in G_{r}$, then $D_{m_{r}}(x)=m_{r}$ and $\chi_{m_{r}}(x)=\exp \left(2 \pi i \alpha(x) / p_{r+1}\right)$, where $\alpha(x) \in\left\{0,1, \cdots, p_{r+1}-1\right\}$ and $\alpha(x)=0$ iff $x \in G_{r+1}$;

(ii) if $x \notin G_{r}$, then $D_{m_{r}}(x)=0$;

(iii) if $x \in G_{r}$, then $\chi_{m_{i}}(x)=1$ for $0 \leqq i<r$.

Since $x \in G$ implies $x \in G_{r} \backslash G_{r+1}$ for some $r$, we have

$$
\begin{aligned}
\left|B_{1}\right| & =\left|\sum_{k=0}^{s-1} \frac{(-1)^{k}}{m_{k+1}-m_{k}} \sum_{j=1}^{p_{k+1}-1} \chi_{m_{k}}^{j}(x) D_{m_{k}}(x)\right| \\
& =\left|\sum_{k=0}^{r} \frac{(-1)^{k}}{m_{k+1}-m_{k}} m_{k} \sum_{j=1}^{p_{k+1}-1} \chi_{m_{k}}^{j}(x)\right| \\
& \leqq\left|\sum_{k=0}^{r-1} \frac{(-1)^{k}}{p_{k+1}-1}\left(p_{k+1}-1\right)\right|+\left|\frac{(-1)^{r}}{p_{r+1}-1}(-1)\right| \\
& =\left|\sum_{k=0}^{r-1}(-1)^{k}\right|+\left|\frac{1}{p_{r+1}-1}\right| \leqq 2 .
\end{aligned}
$$

For $B_{2}$ we have

$$
\left|B_{2}\right| \leqq \frac{1}{m_{s+1}-m_{s}} \sum_{i=m_{s}}^{m-1}\left|\chi_{i}(x)\right| \leqq \frac{m-m_{s}}{m_{s+1}-m_{s}} \leqq 1 .
$$


Consequently, $\left|S_{m}(x ; S)\right| \leqq 4$, uniformly in $x \in G$ and for all $m$.

In the next lemma we define the Fejér polynomials $Q_{n}(x)$ in $X$. The definition is the same as that given by F. Schipp [3] for the case of the Walsh functions.

LEMMA 2. For each $n$, let $\mu_{n}=\sum_{k=0}^{n-1} z_{k} m_{k}$, with $z_{k}=0$ if $k$ is odd and $z_{k}=1$ if $k$ is even. Let

$$
Q_{n}(x)=\chi_{\mu_{n}}(x) \sum_{k=0}^{m_{n}-1} a_{k} \chi_{k}(x) .
$$

Then

(i) $S_{\mu_{n}}\left(0 ; Q_{n}\right)>(n-2) / 2 p$ and

(ii) $\left|Q_{n}(x)\right| \leqq 4(x \in G)$.

Proof. For any two nonnegative integers $k$ and $l$, let $k \oplus l$ be defined by the property that $\chi_{k}(x) \chi_{l}(x)=\chi_{k \oplus l}(x)$. Then, clearly, the Fourier series of $Q_{n}(x)$ is the series

$$
\sum_{k=0}^{m_{n}-1} a_{k} \chi_{k \oplus \mu_{n}}(x) .
$$

Also, since for each $s, \chi_{m_{s}}^{p_{s+1}}=\chi_{t}$ for some $t<m_{s}[4,2.2]$, it follows that if $k$ is of the form $k=\left(p_{28+1}-1\right) m_{2 s}+k^{\prime}$ with $0 \leqq k^{\prime}<m_{2 s}$ and some $s \in\{0,1, \cdots,[(n-1) / 2]\}$, then $a_{k}=\left(m_{2 s+1}-m_{2 s}\right)^{-1}$ and $k \oplus \mu_{n}<\mu_{n}$.

Moreover, these are the only values of $k, 0 \leqq k<m_{n}$, for which $k \oplus \mu_{n}<\mu_{n}$. Consequently,

$$
\begin{aligned}
S_{\mu_{n}}\left(0 ; Q_{n}\right) & =\sum_{s=0}^{[(n-1) / 2]} \frac{1}{m_{2 s+1}-m_{2 s}} m_{2 s}=\sum_{s=0}^{[(n-1) / 2]} \frac{1}{p_{2 s+1}-1} \\
& >\frac{1}{p}\left[\frac{n-1}{2}\right] \geqq \frac{n-2}{2 p} .
\end{aligned}
$$

The second inequality of Lemma 2 follows immediately from Lemma 1.

Proof of The Theorem. Let the function $f$ be defined by

$$
f(x)=\sum_{k=1}^{\infty} \frac{1}{k^{\prime}} \chi_{m_{k^{\prime}}}(x) Q_{k^{\prime}}(x),
$$

where we write $k^{\prime}$ for $2^{k}$. Since for each $k$ the Fourier series of $\chi_{m_{k^{\prime}}}(x) Q_{k^{\prime}}(x)$ contains only characters $\chi_{s}$ with $m_{k^{\prime}} \leqq s<2 m_{k^{\prime}}<m_{(k+1)^{\prime}}$, different values of $k$ correspond to different sets of characters in $X$. Consequently, Lemma 2(i) implies that, for each $k>1$, 


$$
\begin{aligned}
\left|S_{m_{k^{\prime}}+\mu_{k^{\prime}}}(0 ; f)-S_{m_{k^{\prime}}}(0 ; f)\right| & =\left|\frac{1}{k^{\prime}} S_{\mu_{k^{\prime}}}\left(0 ; Q_{k^{\prime}}\right)\right| \\
& >\frac{1}{k^{\prime}} \cdot \frac{k^{\prime}-2}{2 p}=\frac{1}{2 p}-\frac{1}{p k^{\prime}} \geqq \frac{1}{4 p} .
\end{aligned}
$$

Therefore, the Fourier series of $f$ does not converge at $0 \in G$.

Next we prove part (ii) of the theorem. Take any natural number $l$ and let $y \in G_{l}$. Choose $\tilde{s}$ so that, for all $k \leqq \tilde{s}$, the Fourier series of $\chi_{m_{k^{\prime}}}(x) Q_{k^{\prime}}(x)$ contains only characters $\chi_{t}(x)$ with $t<m_{l}$, whereas the Fourier series of $\chi_{m_{(\tilde{s}+1)^{\prime}}}(x) Q_{(\tilde{s}+1)^{\prime}}(x)$ contains at least one $\chi_{t}(x)$ with $t \geqq m_{l}$. Then $m_{(\bar{s}+2)^{\prime}} \geqq m_{l}$, i.e., $2^{-(\bar{s}+2)} \leqq l^{-1}$. Also, since $y \in G_{l}, \chi_{t}(x+y)$ $=\chi_{t}(x)$ for all $x \in G$ and $0 \leqq t<m_{l}$. Therefore,

$$
\begin{aligned}
|f(x+y)-f(x)| \leqq & \left|\sum_{k=1}^{\delta} \frac{1}{k^{\prime}}\left[\chi_{m_{k^{\prime}}}(x+y) Q_{k^{\prime}}(x+y)-\chi_{m_{k^{\prime}}}(x) Q_{k^{\prime}}(x)\right]\right| \\
& +\sum_{k=\tilde{s}+1}^{\infty} \frac{1}{k^{\prime}}\left|\chi_{m_{k^{\prime}}}(x+y)\right|\left|Q_{k^{\prime}}(x+y)\right| \\
& +\sum_{k=\tilde{s}+1}^{\infty} \frac{1}{k^{\prime}}\left|\chi_{m_{k^{\prime}}}(x)\right|\left|Q_{k^{\prime}}(x)\right| \\
\leqq & +\sum_{k=\tilde{s}+1}^{\infty} \frac{1}{k^{\prime}}\left|Q_{k^{\prime}}(x+y)\right|+\sum_{k=\delta+1}^{\infty} \frac{1}{k^{\prime}}\left|Q_{k^{\prime}}(x)\right| .
\end{aligned}
$$

Lemma 2(ii) implies that

$$
|f(x+y)-f(x)| \leqq 2 \cdot 4 \sum_{k=\overline{8}+1}^{\infty} \frac{1}{2^{k}}=8 \frac{1}{2^{\bar{s}}}=O\left(\frac{1}{l}\right) .
$$

Hence $\theta_{l}(f)=O\left(l^{-1}\right)$. This completes the proof of the theorem.

\section{REFERENCES}

1. N. J. Fine, On the Walsh functions, Trans. Amer. Math. Soc. 65 (1949), 372-414. MR 11, 352.

2. C. W. Onneweer and Daniel Waterman, On uniform convergence of Fourier series on groups. I, Michigan Math. J. (to appear).

3. F. Schipp, Bemerkung zur Divergenz der Walsh-Fourierreihen, Ann. Univ. Sci. Budapest. Eötvös Sect. Math. 11 (1968), 53-58. MR 39 \#3226.

4. N. Ja. Vilenkin, On a class of complete orthonormal systems, Izv. Akad. Nauk SSSR Ser. Mat. 11 (1947), 363-400; English transl., Amer. Math. Soc. Transl. (2) 28 (1963), 1-35. MR 9, 224; MR 27 \#4001.

5. A. Zygmund, Trigonometric series. Vol. 1, 2nd ed., Cambridge Univ. Press, New York, 1959. MR 21 \#6498.

University of New Mexico, Albuquerque, New Mexico 87106 